\title{
Properties of X-ray binaries in the Magellanic Clouds from $R X T E$ and Chandra observations
}

\author{
R. H. D. Corbet ${ }^{1,2}$, M. J. Coe ${ }^{3}$, K. E. McGowan ${ }^{3}$, M. P. E. Schurch ${ }^{3}$, \\ L. J. Townsend ${ }^{3}$, J. L. Galache ${ }^{4}$ and F. E. Marshall ${ }^{2}$ \\ ${ }^{1}$ University of Maryland, Baltimore County/CRESST, 1000 Hilltop Circle, Baltimore, \\ MD 21250, USA \\ ${ }^{2}$ NASA Goddard Space Flight Center, Greenbelt, MD 20771, USA \\ ${ }^{3}$ School of Physics and Astronomy, University of Southampton, SO17 1BJ, UK \\ ${ }^{4}$ Harvard-Smithsonian Center for Astrophysics, 60 Garden Street, Cambridge, MA 02138, USA
}

\begin{abstract}
The X-ray binary population of the SMC is very different from that of the Milky Way consisting, with one exception, entirely of transient pulsating Be/neutron star binaries. We have now been monitoring these SMC X-ray pulsars for over 10 years using the Rossi X-ray Timing Explorer with observations typically every week. The RXTE observations have been complemented with surveys made using the Chandra observatory. The RXTE observations are non-imaging but enable detailed studies of pulsing sources. In contrast, Chandra observations can provide precise source locations and detections of sources at lower flux levels, but do not provide the same timing information or the extended duration light curves that RXTE observations do. We summarize the results of these monitoring programs which provide insights into both the differences between the SMC and the Milky Way, and the details of the accretion processes in X-ray pulsars.
\end{abstract}

Keywords. stars: emission-line, Be, stars: neutron, pulsars: general, Magellanic Clouds, X-rays: binaries

\section{Introduction}

Mass transfer in high-mass X-ray binaries (HMXBs) may occur in 3 different ways from the OB star component. (i) The mass-donor primary star may fill its Roche lobe. These systems are very luminous $\left(\sim 10^{38} \mathrm{erg} \mathrm{s}^{-1}\right)$ but are very rare. (ii) If the system contains a supergiant primary with an extensive stellar wind then accretion from the wind may take place. These systems have modest luminosity $\left(\sim 10^{36-37} \mathrm{erg} \mathrm{s}^{-1}\right)$ but are rather more common. (iii) For systems containing a Be star accretion takes place from the circumstellar envelope. These have a wide range of luminosities $\left(10^{34-39} \mathrm{erg} \mathrm{s}^{-1}\right)$ and are very common, but are transient.

In most HMXBs the accreting object is a highly magnetized neutron star. Accretion is funneled onto the magnetic poles of the neutron star and we see pulsations at the neutron star spin period. If the pulse periods of HMXBs are plotted against their corresponding orbital periods then it is seen that sources divide into three groups in this diagram which correspond to the three modes of mass transfer (Corbet 1986). In particular there is strong correlation between pulse period and orbital period for the Be star systems. The positions of sources in this diagram is thought to depend on the accretion torques experienced by the neutron stars and hence on the circumstellar environments around the primary stars. These classes of HMXB are well-studied in the Galaxy and we wish to 


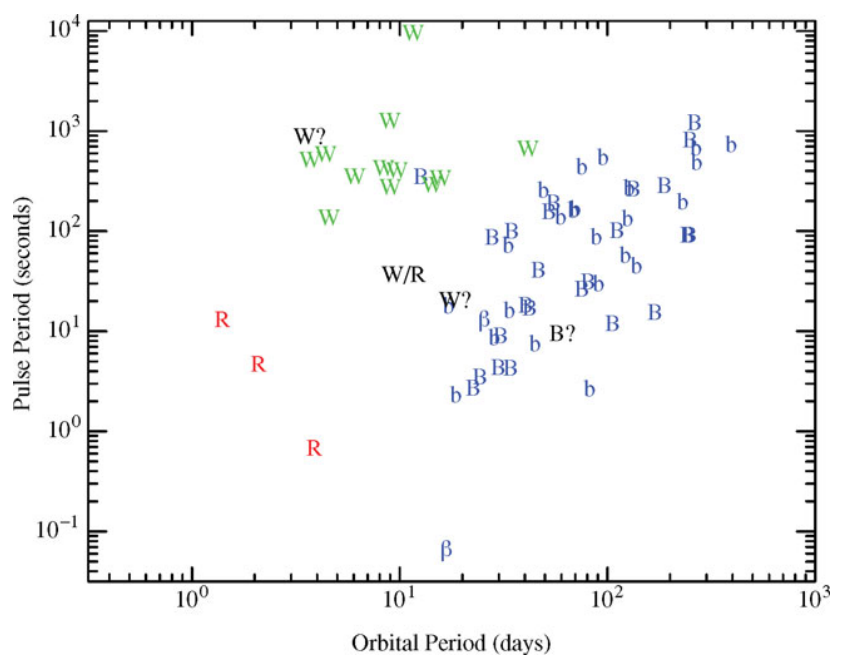

Figure 1. Pulse period vs. orbital period for HMXBs. "R" = Roche lobe overflow, "W" = wind accretion, "B" = Galactic Be star source, "b" = SMC Be star source, " $\beta "$ = LMC Be star source.

know how the HXMB populations compare in other galaxies. Because of their proximity, the SMC and LMC make them the easiest external galaxies to investigate.

Initial estimates of the HMXB population of the SMC were based on the mass of the SMC. The SMC mass is a few percent of the mass of the Galaxy and about 65 Galactic X-ray pulsars are known. Therefore, 1 or 2 X-ray pulsars would be expected in the SMC. The larger fraction of Be stars in the SMC increased the estimate to $\sim 3$. The first X-ray pulsar discovered in the SMC was SMC X-1 in 1970s. Its luminosity can reach $\sim 10^{39}$ erg $\mathrm{s}^{-1}$ and it has a $0.71 \mathrm{~s}$ pulse period and a 3.89 day orbital period. The mass-donating companion is a Roche-lobe filling B0I star. In 1978 two transients, SMC X-2 and SMC X3, were found (Clark et al. 1978). The three pulsars then known agreed with the simple prediction, although all three were surprisingly bright.

\section{2. $R X T E$ observations of the SMC}

$R X T E$ was launched in 1995 and its primary instrument is the Proportional Counter Array (PCA). The RXTE PCA has a $2^{\circ} \mathrm{FWZI}, 1^{\circ} \mathrm{FWHM}$ field of view. The PCA is non-imaging, but it has a large collecting area of up to $7,000 \mathrm{~cm}^{2}$. The $R X T E$ observing program is extremely flexible and almost all observations are time constrained. These include monitoring, phase constrained, and target of opportunity observations as well as observations coordinated with other observatories both in space and ground-based.

Serendipitous $R X T E$ PCA slew observations in 1997 showed a possible outburst from SMC X-3 (Marshall et al. 1997). A follow-up pointed RXTE observation showed a complicated power spectrum with several harmonic, almost-harmonic, and non-harmonic peaks. Imaging $A S C A$ observations were then made of this region and they showed the presence of two separate pulsars. However, neither of these pulsars coincided with the position of SMCX-3. A revised look at the $R X T E$ power spectrum revealed three pulsars simultaneously active with periods of 46.6, 91.1, and $74.8 \mathrm{~s}$ (Corbet et al. 1998).

Since 1997 we have monitored one or more positions weekly using the $R X T E$ PCA. The flexible observing program of $R X T E$ has enabled us to carry out a regular monitoring 


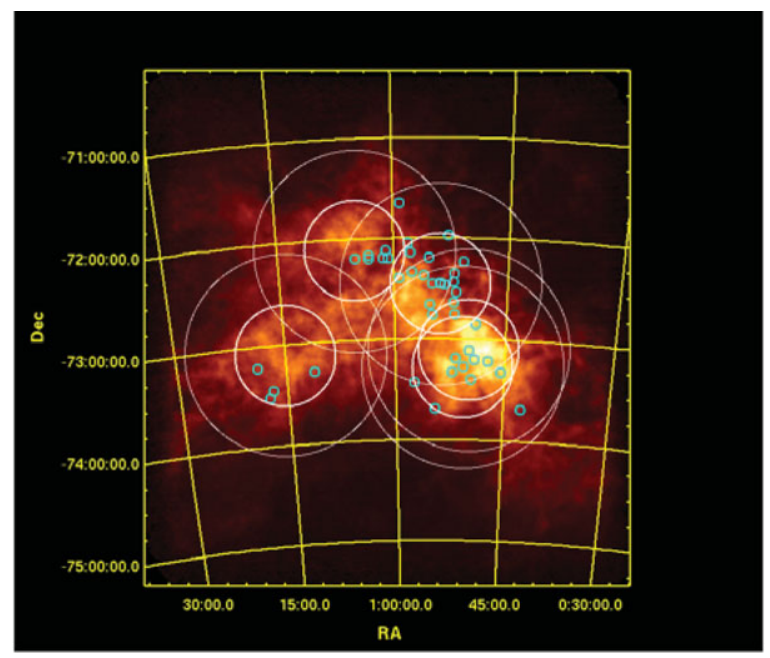

Figure 2. H I Image of the SMC. Large circles = PCA FOV (FWHM and FWZI) at different monitoring positions. Small circles show locations of X-ray pulsars.

program that would not have been possible with other satellites. The typical observation duration has been about 10,000 seconds. We use power spectra of the light curves to extract pulsed flux from any X-ray pulsars in the FOV. The sensitivity to pulsed flux is $\sim 10^{36} \mathrm{erg} \mathrm{s}^{-1}$ at the distance of the SMC. From this program we have detected many transient sources and all identified optical counterparts have been found to be Be stars. The SMC HMXB pulsar population has now been found by ourselves and other investigators to be much larger than originally thought. Our naming convention for SMC pulsars is SXPx, where "x" is the pulse period, for SMC X-ray Pulsar. This convention is particularly useful for X-ray pulsars discovered with $R X T E$ for which a precise position is not yet available. For detailed light curves and analyses see Laycock et al. (2005) and Galache et al. (2008). In addition, we have recently been able to measure orbital parameters from Doppler modulation of the pulse period of SXP 18.3 (Schurch et al. 2008).

The Be pulsar spin period/orbital period correlation is believed to be related to the structure of the extended envelopes of Be stars. SMC and Milky Way Be stars have differences, for example, the SMC metallicity is far lower and the Be phenomenon is more common in the SMC. Is this reflected in the $\mathrm{P}_{\mathrm{S}} / \mathrm{P}_{\text {orb }}$ relation? That is, are there significant differences between Be star envelopes in the SMC and the Galaxy? For a linear fit (to the log- log diagram) the intercept is related to Be star mass loss rates and the gradient is related to the radial structure of Be star envelopes.

Currently 23 SMC Be X-ray pulsars now have measured orbital periods. The periods have been measured by several techniques. These include: X-ray flux monitoring with $R X T E$, pulse timing with $R X T E$ (one system) and optical observations from MACHO and OGLE. In comparison, 24 Galactic Be X-ray pulsars now have measured orbital periods. We find that for the SMC and Galactic systems the intercepts are the same, the gradients are the same, and the scatter about the fits are the same. Thus, the metallicity difference between the two galaxies gives no measurable effect on the spin period/orbital period relationship and the Be star envelopes in SMC and Galaxy are apparently similar.

Galache (2006) proposes that the frequency of outbursts per orbit (X-ray "outburst density" or $\mathrm{X}_{\mathrm{od}}$ ) depends on the orbital period. Long period systems are more likely to show an outburst at periastron. The reason for this correlation is not yet clear. 


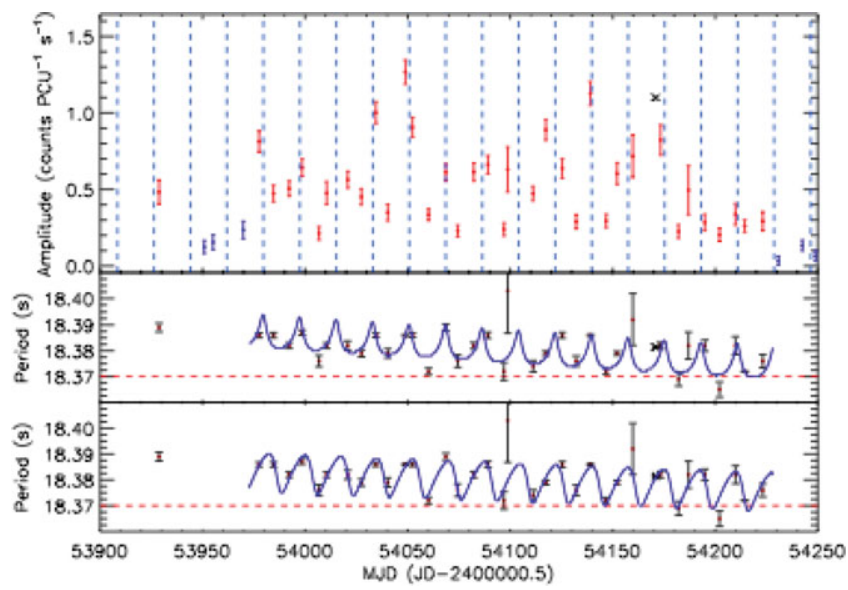

Figure 3. The extended outburst from SXP 18.3. The top panel shows the amplitude of the pulsed flux. The two lower panels show two possible timing solutions. The middle panel shows the preferred solution with the orbital period fixed at the photometric period. (Schurch et al. 2009).
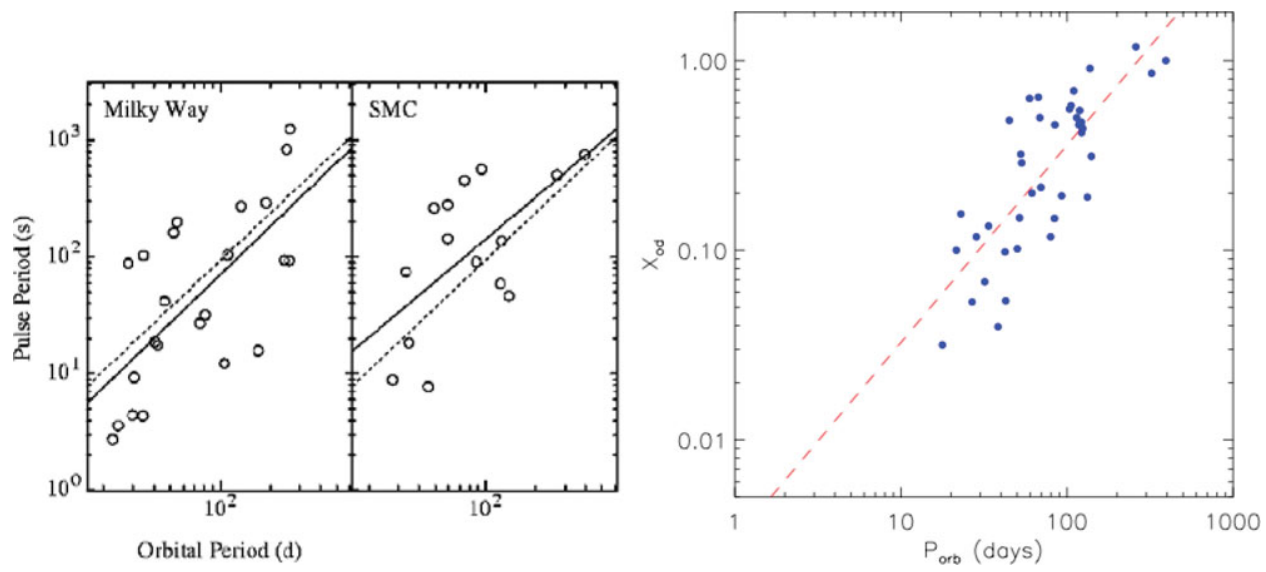

Figure 4. Left: A comparison of the $\mathrm{P}_{\mathrm{s}} / \mathrm{P}_{\text {orb }}$ relationships for the SMC and the Galaxy. Right: The relationship between outburst density and orbital period proposed by Galache (2006).

\section{Chandra SMC Wing survey}

A possible connection between hydrogen column density $\left(\mathrm{N}_{\mathrm{H}}\right)$ and HMXB location was proposed by Coe et al. (2005). To investigate this we undertook a survey of the SMC wing using Chandra. We observed 20 fields with $\sim 10$ ks observation time per field. 523 sources were detected (McGowan et al. 2008a), but only $\sim 5$ of these were HMXBs (McGowan et al. 2007) and the majority of sources are probably background AGNs. There thus appear to be fewer X-ray pulsars in the wing than the bar. This is despite that fact that the most luminous SMC HMXB, SMC X-1 is located in the wing. 

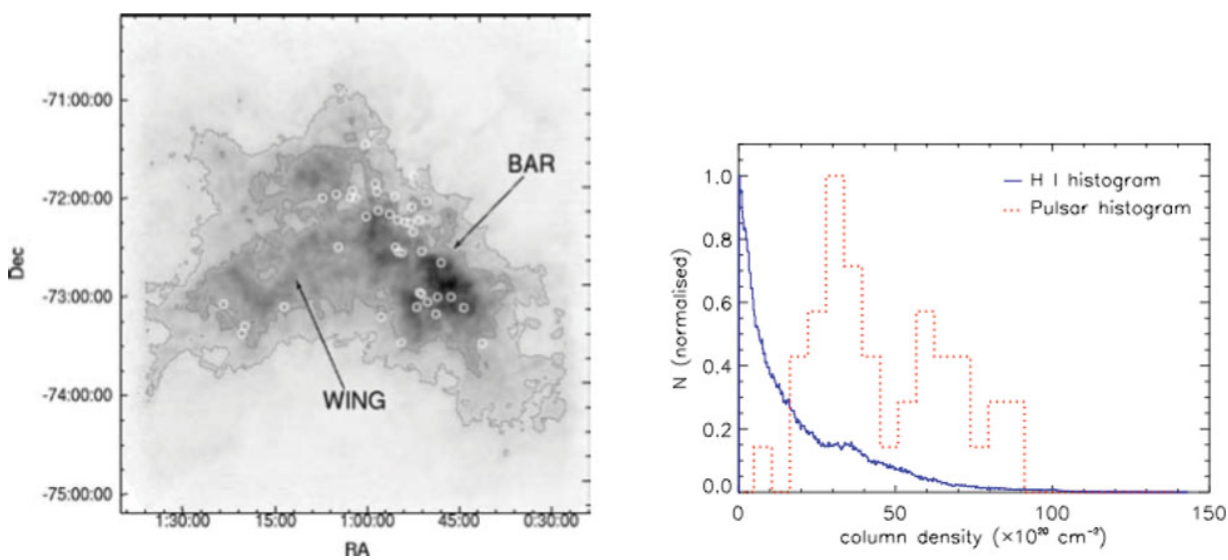

Figure 5. Left: The location of SMC pulsars superimposed on an H I contour map. Right: Histogram of SMC H I distributions and corresponding histogram of H I columns at the location of the X-ray pulsars (Coe et al. 2005).

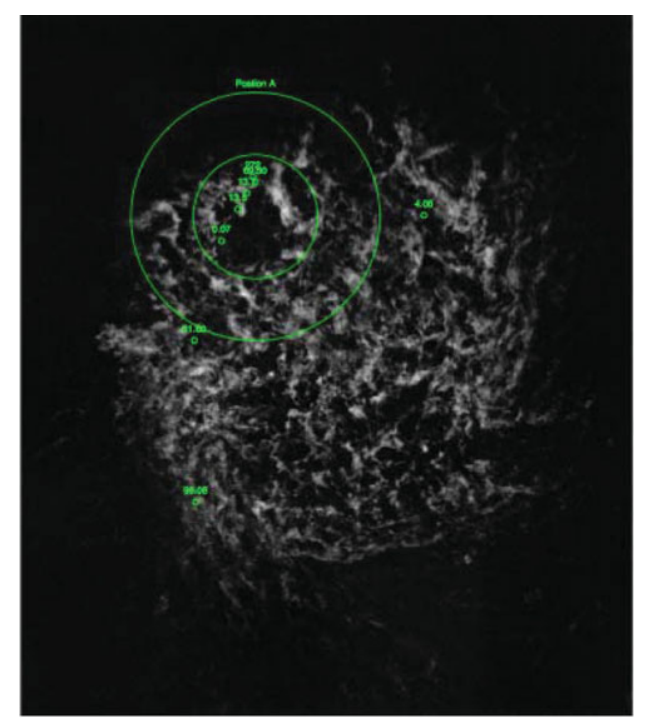

Figure 6. The PCA monitoring position for the LMC. Known pulsar positions are marked.

\section{4. $R X T E$ monitoring of the LMC}

The SMC appears to be very abundant in Be X-ray pulsars. This was only known after regular observations of the SMC started. The known LMC X-ray pulsar population is more modest. There is one Roche lobe overflow source, and a few Be systems. To investigate the LMC population in more detail we undertook an $R X T E$ monitoring program similar to the one used for the SMC. However, the angular size of the LMC is larger so we restricted the program to monitoring one position that was already know to contain several X-ray sources. We analyzed data from our one year monitoring program, together with archival data from other programs (Townsend et al., in preparation). In the monitoring region 4 of the 5 known X-ray pulsars were detected. However, no new $\mathrm{X}$-ray pulsars were discovered. This implies that the X-ray pulsar content of the LMC is more like that of the Galaxy than the SMC. 


\section{Conclusion}

The current census of SMC X-ray pulsars is: 1 supergiant Roche lobe filler (SMCX-1); $\sim 50$ transients (likely all Be star systems); 1 possible Crab-like pulsar $(P=0.087 \mathrm{~s})$ from $A S C A$ (Yokogawa \& Koyama 2000); 1 Anomalous X-ray Pulsar (AXP) candidate $(P=8.02 \mathrm{~s})$ from Chandra and $X M M$; no supergiant wind accretion systems and no low-mass X-ray binaries. Supergiant wind systems should easily be detectable at our $\sim 10^{36} \mathrm{erg} \mathrm{s}^{-1}$ pulsed flux sensitivity. An obvious question is: why are there so many SMC X-ray pulsars? The current star formation rate in the SMC is reported not to be extremely high. The lifetime of HMXBs is short which implies an enhanced star formation rate in the recent past. However, supergiant wind systems, which have even shorter lifetimes than Be star systems, have not been found. Models of historic star formation rates in the SMC and LMC must be compatible with the observed X-ray binary populations, and they most also account for the differences between the SMC and LMC.

There are also similarities between the SMC and Galactic pulsar populations. The SMC and Galactic Be star systems have identical (within errors) $\mathrm{P}_{\mathrm{S}} / \mathrm{P}_{\text {orb }}$ relationships. The LMC X-ray pulsar population also appears to be more similar to that of the Galaxy. The large and growing SMC X-ray pulsar database has considerable potential for understanding the astrophysics of accretion processes. It facilitates comparative studies, such as pulse profile morphology, as a function of luminosity. Or, luminosity effects can be removed and we can examine the effects of other parameters such as magnetic field strength. The SMC is nearby and optical counterparts can be observed with modest size telescopes. In particular, MACHO and OGLE lightcurves exist for many counterparts (e.g., Coe et al. this volume; McGowan et al. 2008b). The overall X-ray pulsar properties can tell us about the evolutionary similarities and differences of a very nearby galaxy compared to our own.

\section{References}

Clark, G., Doxsey, R., Li, F., Jernigan, J. G., \& van Paradijs, J. 1978, ApJ, 221, L37

Coe, M. J., Edge, W. R. T., Galache, J. L., \& McBride, V. A. 2005, MNRAS, 356, 502

Corbet, R. H. D. 1986, MNRAS, 220, 1047

Corbet, R. H. D., Marshall, F. E., Lochner, J. C., Ozaki, M., \& Ueda, Y. 1998, IAUC, 6803

Galache, J. L. 2006, PhD thesis, University of Southampton

Galache, J. L., Corbet, R. H. D., Coe, M. J., Laycock, S., Schurch, M. P. E., Markwardt, C., Marshall, F. E., \& Lochner, J. 2008, ApJS, 177, 189

Laycock, S., Corbet, R. H. D., Coe, M. J., Marshall, F. E., Markwardt, C., \& Lochner, J. 2005, ApJS, 161, 96

Marshall, F. E., Lochner, J. C., \& Takeshima, T. 1997, IAUC, 6777

McGowan, K. E., Coe, M. J., Schurch, M. P. E., et al. 2007, MNRAS, 376, 759

McGowan, K. E., Coe, M. J., Schurch, M. P. E., et al. 2008a, MNRAS, 383, 330

McGowan, K. E., Coe, M. J., Schurch, M. P. E., Corbet, R. H. D., Galache, J. L., \& Udalski, A. 2008b, MNRAS, 384, 821

Schurch, M. P. E., Coe, M. J., Galache, J. L., et al. 2009, MNRAS, 392, 361

Yokogawa, J. \& Koyama, K. 2000, in D. W. E. Green (ed.) IAUC, 7361 